Descargo de responsabilidad: las opiniones expresadas en los manuscritos son responsabilidad exclusiva de los autores. No necesariamente reflejan las opiniones de la editorial ni la de sus miembros.

$1 \mathrm{CENIRO}$ NIVERSITARIO DEACAPA

\title{
Suicidio y manifestaciones psicológicas frente al covid-19 en adolescentes de Camotán
}

Suicide and psychological manifestations against covid-19 in an adolescent from Camotan

Recibido: $12 / 10 / 2021$

Publicado: 20/01/2022
Kateryn Morelia Mejia Betancourth

Maestría en Neurociencias con enfasis en neurocognición

Universidad de San Carlos de Guatemala

kmoreliaamejia@gmail.com

https://orcid.org/0000-0002-7310-5167

\section{Referencia}

Mejia Betancourth , K. M. (2022). Suicidio y manifestaciones psicológicas frente al covid-19 en adolescentes de Camotán. Revista Académica Sociedad Del Conocimiento Cunzac, 2(1), 9-15.

DOI: https://doi.org/10.46780/sociedadcunzac.v2i1.7

\section{Resumen}

OBJETIVO: determinar la intencionalidad suicida durante pandemia COVID-19 en adolescentes de secundaria del municipio de Camotán MÉTODO: se realizó un estudio descriptivo transversal en 80 adolescentes del nivel secundario del municipio de Camotán. RESULTADOS: se determinó que el 35.1\% $(\mathrm{N}=28)$ de los adolescentes pertenecen a la edad de 14 años, los factores que provocan la ideación suicida están los de mayor prevalencia de tipo personal como lo es la depresión, estrés y ansiedad con un $40 \%, \quad(\mathrm{~N}=32)$ los datos obtenidos a través de la escala de ideación suicida muestra que la población de estudio presenta débil deseo de vivir con un total de $67.5 \%$, ( $\mathrm{N}=54)$. CONCLUSION: el suicidio es un fenómeno multifactorial y un problema de mucha relevancia de salud pública por lo cual el elemento frágil como consecuencia para la ideación suicida está el temor a fallecer por COVID-19 obteniendo un total de $38.8 \%(\mathrm{~N}=31)$ de las respuestas de los adolescentes de secundaria del municipio de Camotán, Chiquimula.

\section{Palabras clave}

suicidio, COVID-19, adolescentes

\section{Abstract}

OBJECTIVE: to evaluate the intentionality, determine the risk factors and describe the fragile elements as a consequence of suicidal ideation during a COVID-19 pandemic in high school adolescents in the municipality of Camotán. METHOD: a cross-sectional descriptive study was carried out in 80 adolescents from the secondary level of the municipality of Camotán. RESULTS: it was determined that $35.1 \%(\mathrm{~N}=28)$ of the adolescents belonged to the age of 14 , the factors that provoke suicidal ideation are those with the highest prevalence of a personal natu- 
re, such as depression, stress and anxiety with a $40 \%,(\mathrm{~N}=32)$ the data obtained through the suicidal ideation scale created by Beck, shows that the study population has a weak desire to live with a total of $67.5 \%,(\mathrm{~N}=54)$. CONCLUSION: Suicidal behavior is a multifactorial phenomenon and an important public health problem, for which the fragile element as a consequence for suicidal ideation is the fear of dying from COVID-19, obtaining a total of $38.8 \%(\mathrm{~N}=31)$ of the responses of adolescents from secondary school in the municipality of Camotán, Chiquimula.

\section{Keywords}

suicide, COVID-19, teens

\section{Introducción}

Según la OMS (2006) el suicidio es comprendido como un trastorno mental multidimensional, resultado de una interacción compleja de diversos factores; genéticos, biológicos, psicológicos y ambientales. Antecedentes de estudios e investigaciones han demostrado la relevancia de las ideas suicidas en adolescentes como una problemática que va en aumento en la sociedad. El suicidio es un problema de salud, que se ha crecido significativamente en el mundo, cada año se suicidan alrededor de 800 mil personas; en otras palabras, cada 40 segundos muere una persona en el mundo por esta causa (OMS, 2006).

Estudios han encontrado un aumento en las tasas de diagnósticos de tipo psicológico durante la pandemia. Ansiedad, alteraciones del sueño y depresión, que pueden manifestarse como comorbilidad, es importante tener en cuenta los sujetos con más alto riesgo de presentar afecciones en su salud mental, pacientes que han contraído la infección, familiares preocupados, personas con comorbilidades psiquiátricas o enfermedades crónicas ya existentes (Nicolini, 2020).

El suicidio es un fenómeno que se ha estudiado en adolescentes en todos los países del mundo desde el punto de vista social, cultural y económico. El incremento del suicidio en los adolescentes se ha convertido en un problema de Salud pública (Martín-del-Campo et al., 2013).

El suicidio es multifactorial, por lo cual, en la consumación del acto suicida, se toman en cuenta factores neurobiológicos, genéticos, psicopatológicos, de interacción familiar y psicosociales. Es decir, una acción multideterminada por diversos factores intervinientes que se potencian, de esta forma se reconoce que la mayoría de los sujetos suicidas han enfrentado procesos negativos que no logran resolver por sí solo (González, et al., 2021).

Existen diversas repercusiones de factores que se asocian al Covid-19 y el suicidio es por ello que es importante generar alertas alrededor del mundo, donde se advierta del probable incremento en la conducta suicida durante la crisis sanitaria incluso una vez que ésta haya sido controlada.

En Guatemala el suicidio es considerado uno de los principales problemas y causas de muerte de jóvenes, especialmente del área rural. Tomando en cuenta la ideación suicida como una etapa temprana del mismo, la cual se manifiesta como ideas o pensamientos autodestructivos, así como la planificación de cómo llevarlo a cabo. 
Si no existe un tratamiento psiquiátrico eficaz pueden cometer un intento de suicidio o un suicidio consumado a un adolescente. Según la PDH, Chiquimula es el departamento en donde más suicidios en adolescentes se han reportado. (Gutiérrez, et al., 2014).

Se tienen como objetivos evaluar la intencionalidad suicida, determinar los factores de riesgo como tambien describir los elementos frágiles como consecuencia de la ideación en adolescentes del nivel secundario del municipio de Camotán, Chiquimula mediante la escala de ideación suicida (Scale for Suicide Ideation, SSI) creada por Beck.

La Escala de Ideación Suicida, es de diseño heteroaplicado, elaborada por Beck para cuantificar y evaluar el intento suicido, el grado de seriedad e intensidad con el que alguien pensó o está pensando suicidarse.

\section{Materiales y métodos}

Estudio descriptivo transversal sobre la idea suicida realizado en adolescentes de secundaria del municipio de Camotán, Chiquimula con una $(\mathrm{N}=80)$, fueron establecidas variables categoricas tales como: edad, sexo y estado civil, las variables independientes fueron los factores que determinan la idea suicida y los elementos frágiles como consecuencia de la misma, para su ejecución se llevó acabo por medio de una boleta de recolección de datos para obtener fácil distribución de la información, así como también la identificación de los factores, distribuyendolos en, familiares, sociales y personales, así mismo los elementos frágiles con relación al COVID-19.

Fue aplicada la escala de ideación suicida de Beck formada por 19 interrogantes para identificar la ideación y la frecuencia del mismo. Estudios sobre fiabilidad realizado por autores sobre muestras se han obtenido una consistencia interna alta y una fiabilidad inter examinador, por lo cual investigaciones anteriores apoyan la validez de constructo, validez concurrente y su capacidad discriminativa.

\section{Resultados}

De acuerdo al estudio realizado y utilizando la boleta de recolección de datos como instrumento de investigación se obtuvieron los siguientes resultados que se representan en las siguientes tablas. 
Tabla 1. Factores que han hecho que presente ideación suicida.

\begin{tabular}{lcc}
\hline $\begin{array}{c}\text { Factores familiares que } \\
\text { determinan la ideación suicida }\end{array}$ & Cantidades & Porcentajes \\
\hline Estudios académicos interrumpidos & 9 & $11.2 \%$ \\
\hline Desgaste emocional & 7 & $8.8 \%$ \\
\hline Descenso de ingresos económicos & 6 & $7.5 \%$ \\
\hline OTROS & 5 & $6.2 \%$ \\
\hline $\begin{array}{l}\text { No tener contacto con las demás } \\
\text { personas }\end{array}$ & 4 & $1.3 \%$ \\
\hline $\begin{array}{l}\text { Violencia Intrafamiliar } \\
\text { Factores sociales determinantes en la }\end{array}$ & 1 & \\
$\begin{array}{l}\text { ideación suicida } \\
\text { Falta de apoyo social personales }\end{array}$ & 4 & $5 \%$ \\
\hline $\begin{array}{l}\text { Factores } \\
\text { determinan la ideación suicida }\end{array}$ & & \\
\hline Depresión, estrés y ansiedad & 32 & $40 \%$ \\
\hline $\begin{array}{l}\text { Baja autoestima } \\
\text { Intentos de suicidio anteriores }\end{array}$ & 0 & $15 \%$ \\
\hline
\end{tabular}

Fuente: elaboración propia. Información extraída de boleta de recolección de datos.

Los resultados obtenidos de los factores que han hecho que los adolescentes del municipio de Camotán presenten ideación suicida están los de mayor prevalencia de tipo personal como lo es la depresión, estrés y ansiedad con un $40 \%(\mathrm{~N}=32)$ mientras 12 de los adolescentes dijeron tener baja autoestima lo que representa un $15 \%$ de la población.

NOTA: dentro de los factores familiares en la casilla OTROS los participantes dijeron: No tener deseo de suicidarse ni han tenido ideación suicida con un total de $2.4 \%(\mathrm{~N}=2)$ y el $3.8 \%$ $(\mathrm{N}=3)$ solo marco la casilla de factores familiares.

Tabla 2. Intencionalidad suicida por medio de la escala de ideación suicida (Scale for Suicide Ideation, SSI) creada por Beck

\begin{tabular}{lcc}
\hline \multicolumn{1}{c}{ ITEMS } & Cantidades & Porcentajes \\
\hline Presenta deseo de vivir & 54 & $67.5 \%$ \\
\hline Débil & 15 & $18.8 \%$ \\
\hline Moderado a fuerte & 11 & $13.7 \%$ \\
\hline Ninguno & & \\
\hline $\begin{array}{l}\text { Dimensión temporal (duración de la } \\
\text { ideación/deseo suicida) }\end{array}$ & 42 & $52.5 \%$ \\
\hline Breve, períodos pasajeros & 32 & $40 \%$ \\
\hline Por amplios periodos de tiempo & 6 & $7.5 \%$ \\
\hline Continuo (crónico) o casi continuo & & \\
\hline $\begin{array}{l}\text { Dimensión temporal (frecuencia del } \\
\text { suicidio) }\end{array}$ & 50 & $62.5 \%$ \\
\hline Intermitente & 27 & $33.8 \%$ \\
\hline Raro, ocasional & 3 & $3.7 \%$ \\
\hline Persistente o continuo & & \\
\hline
\end{tabular}

Fuente: elaboración propia. Información extraída de boleta de recolección de datos. 
Los datos obtenidos a través de la escala de ideación suicida creada por Beck, muestra que la población de estudio presenta débil deseo de vivir con un total de $67.5 \%,(\mathrm{~N}=54)$ la dimension temporal (duración de la ideación) fue breve, con períodos pasajeros obtieniendo el $52.5 \%$, ( $N=42)$ la dimension temporal (frecuencia del suicidio) fue intermitente con un total del $62.5 \%(\mathrm{~N}=50)$.

Tabla 3. Elementos frágiles como consecuencia para la ideación suicida.

\begin{tabular}{lcc}
\hline & Cantidades & Porcentajes \\
\hline Temor a fallecer por COVID & 31 & $38.8 \%$ \\
\hline $\begin{array}{l}\text { Estado de impotencia, tristeza y } \\
\text { soledad por el confinamiento }\end{array}$ & 19 & $23.7 \%$ \\
\hline La perdida de familiares o & 19 & $23.7 \%$ \\
compañeros de estudios a causa de & & \\
COVID-19 & 11 & $13.8 \%$ \\
$\begin{array}{l}\text { Aparecimiento de casos CoVID-19 } \\
\text { alertas rojas en los diferentes } \\
\text { departamentos }\end{array}$ & & \\
\hline
\end{tabular}

Fuente: elaboración propia. Información extraída de boleta de recolección de datos.

Por medio de la tabulación de datos se pudieron constatar los elementos frágiles como consecuencia de que los adolescentes tengan ideación suicida está el temor de fallecer por COVID con un $38.8 \%(\mathrm{~N}=31)$ mientras que el estado de impotencia, tristeza y soledad por el confinamiento lo ocupa el $23.7 \%(\mathrm{~N}=19)$ como también la perdida de familiares o compañeros de estudio a causa del COVID, el elemento frágil era el aparecimiento de casos COVID-19 o alertas rojas en los diferentes departamentos, ocupando el $13.8 \%(\mathrm{~N}=11)$.

\section{Discusión}

Los resultados que se obtuvieron por medio de la boleta de recolección de datos clasificaron las siguientes características sociodemográficas las cuales se describen a continuación: el $35.1 \%$ de los adolescentes pertenecen a la edad de 14 años $(\mathrm{N}=28)$, el sexo predominante de la población fueron las mujeres con un $68.8 \%(\mathrm{~N}=55)$, el estado civil de los participantes fue el $100 \%$ solteros $(\mathrm{N}=80)$. Dentro de los factores personales, la depresión, estrés y ansiedad predomino con un $40 \%(\mathrm{~N}=32)$ la intencionalidad suicida que describen los adolescentes demuestran que el $67.5 \%(\mathrm{~N}=54)$ tiene débil deseo de vivir, la dimensión de frecuencia de la ideación es intermitente con el $62.5 \%(\mathrm{~N}=50)$, el elemento frágil como consecuencia para la ideación suicida está el temor a fallecer por COVID-19 obteniendo un total de $38.8 \%(\mathrm{~N}=31)$ de las respuestas de la población de los adolescentes de secundaria del municipio de Camotán, Chiquimula.

Se realizó una comparación con el estudio sobre ideación suicida, depresión y autoestima en adolescentes escolares de Santa Marta Colombia, el autor publicó un estudio donde buscó dar 
a conocer la relación que existe entre depresión, autoestima e ideación suicida en estudiantes adolescentes de media vocacional en Santa Marta. La evaluación fue realizada a través del Inventario de Depresión de Beck, la Escala de Autoestima de Rosenberg, la Escala de Ideación Suicida de Beck como también un cuestionario socio demográfico. La muestra estuvo conformada por 242 estudiantes entre 15 a 19 años (Media=15 DesvTip=15.3), escogidos a través de un muestreo aleatorio estratificado. Los resultados muestran que el existe relación entre la ideación suicida y depresión $(r=0.295 \mathrm{Sig}=0.00)$, así como con la autoestima baja $(r=-0.209$ Sig=0.00). (Ceballos-Ospino, et al., 2015).

En su estudio destaca la presencia de las variables en estudio obtenidas en esta investigación fueron del $25 \%$ para la ideación suicida entre riesgo medio y alto, $16 \%$ para la depresión y del $40 \%$ para la baja autoestima. De lo anterior, hallado en los resultados, se nota que estadísticamente existe entre depresión e ideación suicida una correlación significativa, lo cual señala que a mayor nivel de depresión presente en lo sujetos, mayor riesgo de ideas suicidas o tienen alta probabilidad que las presenten en algún momento de su vida (Ceballos-Ospino, et al.,2015).

\section{Referencias}

Ceballos-Ospino, G. A., Suarez-Colorado, Y., Suescún-Arregocés, J., Gamarra-Vega, L. M., González, K. E., \& Sotelo-Manjarres A. P. (2015). Ideación suicida, depresión y autoestima en adolescentes escolares de Santa Marta. Rev.Duazary, 12(1), http://revistas.unimagdalena.edu.co/index.php/duazary/article/view/1394

González, S. R., Martínez, C. A., Domínguez, M. W., \& Garcia, R. I., (2021). Un acercamiento al conocimiento de la presencia de marcadores neurobiológicos en la génesis del suicidio. Rev Medica Granma. 25(2), http://scielo.sld.cu/scielo.php?script=sci_arttext\&pid=S1028-48182021000200014\&lng=es\&tlng=es

Gutiérrez, E., Lee, J., Rivera, R., (2014). Intencionalidad Suicida en Pubertad y Adolescencia. (Tesis de Licenciatura) Universidad de San Carlos de Guatemala, Centro Universitario de Oriente, CUNORI. http://cunori.edu.gt/descargas/TESIS_INTENCIONALIDAD_SUICIDA.pdf

Martín-del-Campo, A., González, C. y Bustamante, J. (2013). El suicidio en adolescentes. Adolescent suicide Rev Med Hosp Gen Méx. 76(4):200-209 http://apsmanizales.com/files/3f.-Suicidio-en-adolecentes.pdf

Nicolini, H., (2020). Depresión y ansiedad en los tiempos de la pandemia de COVID-19. Rev Permanyer 88(5), DOI: https://doi.org/10.24875/CIRU.M20000067

Organización Mundial de la Salud, (2006). Prevención del suicidio, recurso para consejeros. Departamento de Salud mental y Abuso de sustancias, trastornos mentales y cerebrales. OMS Ginebra. https://www.who.int/mental_health/media/counsellors_spanish.pdf. 


\section{Sobre la autora}

Kateryn Morelia Mejía Betancourth, tiene cierre de pensum de Licenciatura de Psicología en el Centro Universitario de Zacapa -CUNZAC-, actualmente estudiante de la maestría en neurociencias con énfasis en neurocognición.

\section{Financiamiento de la investigación}

Con recursos propios de los investigadores

\section{Declaración de intereses}

Declaran no tener ningún conflicto de intereses, que puedan haber influido en los resultados obtenidos o las interpretaciones propuestas.

\section{Declaración de consentimiento informado}

El estudio se realizó respetando el Código de ética y buenas prácticas editoriales de publicación.

\section{Derechos de uso}

Copyright@ 2022 por Keteryn Morelia Mejia Betancourth

Este texto está protegido por una licencia Creative Commons 4.0

Este texto está protegido por la Licencia Creative Commons Atribución 4.0 Internacional.

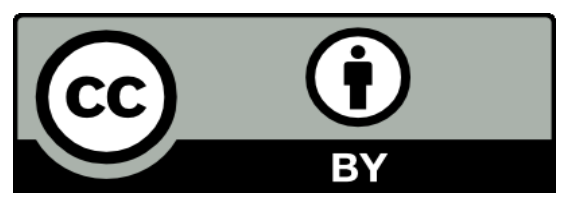

Este texto está protegido por una licencia

Creative Commons 4.0.

Es libre para compartir, copiar y redistribuir el material en cualquier medio o formato y adaptar el documento, remezclar, transformar y crear a partir del material para cualquier propósito, incluso comercialmente, siempre que cumpla la condición de atribución: debe reconocer el crédito de una obra de manera adecuada, proporcionar un enlace a la licencia, e indicar si se han realizado cambios. Puede hacerlo en cualquier forma razonable, pero no de forma tal que sugiera que tiene el apoyo del licenciante o lo recibe por el uso que hace. 